\title{
BMJ Open Randomised trial assessing the impact of framing of fracture risk and osteoporosis treatment benefits in patients undergoing bone densitometry
}

\author{
Rama Kalluru, ${ }^{1,2}$ Keith J Petrie ${ }^{3}$ Andrew Grey, ${ }^{1}$ Zaynah Nisa, ${ }^{1}$ Anne M Horne, ${ }^{1}$ \\ Greg D Gamble, ${ }^{1}$ Mark J Bolland ${ }^{1}$
}

To cite: Kalluru R, Petrie KJ, Grey A, et al. Randomised trial assessing the impact of framing of fracture risk and osteoporosis treatment benefits in patients undergoing bone densitometry. BMJ Open 2017;7:e013703

doi:10.1136/bmjopen-2016013703

- Prepublication history and additional material is available. To view please visit the journal (http://dx.doi.org/ 10.1136/bmjopen-2016013703)

Received 31 July 2016 Revised 5 December 2016 Accepted 23 January 2017

CrossMark

\footnotetext{
${ }^{1}$ Department of Medicine, University of Auckland, Auckland, New Zealand ${ }^{2}$ Department of

Rheumatology, Greenlane Clinical Centre, Auckland, New Zealand

${ }^{3}$ Department of Psychological Medicine, University of Auckland, Auckland, New Zealand
}

Correspondence to Dr Mark Bolland; m.bolland@auckland.ac.nz

\section{ABSTRACT}

Objectives: The accuracy of patients' perception of risk is important for decisions about treatment in many diseases. We framed the risk of fracture and benefits of treatment in different ways and assessed the impact on patients' perception of fracture risk and intentions to take medication.

Design: Randomised trial of 4 different presentations of fracture risk and likely benefits from osteoporosis treatment.

Setting: Academic centre.

Participants: 200 patients undergoing bone densitometry.

Intervention: Presentation that framed the patient's absolute fracture risk either as the chance of having or not having an event, with their likely benefits from osteoporosis treatment in natural frequencies or numbers needed to treat.

Outcomes: Participants' views about their fracture risk and the need for osteoporosis treatment.

Results: The median 5-year fracture risk threshold participants regarded as high enough to consider preventative medication was $50-60 \%$, and did not change substantially after the presentation. The median (Q1, Q3) 5-year risk initially estimated by participants was $20 \%(10,50)$ for any fracture and $19 \%(10,40)$ for hip fracture. $61 \%$ considered their fracture risk was low or very low, and $59-67 \%$ considered their fracture risk was lower than average. These participant estimates were 2-3 times higher than Garvan calculator estimates for any fracture, and 10-20 times higher for hip fracture. Participant estimates of fracture risk halved after the presentation, but remained higher than the Garvan estimates (1.5-2 times for any fracture, 5-10 times for hip fracture). There was no difference in these outcomes between the randomised groups. Participants' intentions about taking medication to prevent fractures were not substantially affected by receiving information about fracture risk and treatment benefits.

Conclusions: Altering the framing of estimated fracture risks and treatment benefits had little effect on participants' perception of the need to take treatment or their individual fracture risk.

Trial registration number: ACTRN12613001081707; Pre-results.

\section{Strengths and limitations of this study}

- A randomised trial assessing different methods of risk communication by investigating the effects of framing of the risk of fracture and benefits of osteoporosis treatments

- Participants were undergoing standard clinical care, so the results may be broadly generalisable.

- Fracture risk was the only risk measurement studied. It is not known whether similar results might be seen in other chronic conditions in which indications for treatment are based on absolute risk such as cardiovascular disease.

- The study cohort was moderately sized and had a relatively low fracture risk.

\section{INTRODUCTION}

Accurate perception of risk is critical for the rational adoption of preventative treatment. People may make decisions about their health based on their perceived risk of future events. Healthcare professionals try to predict the risks of these future events and present this information to patients to assist in making decisions about their treatment. Predictive models have been developed for many conditions, and calculators that integrate data on risk factors to estimate absolute risk for individuals are frequently used. These tools can lead to substantial shifts in disease management. Thus, management of cardiovascular risk has moved from individual risk factors such as blood pressure to become based on absolute cardiovascular risk. ${ }^{1}$ Likewise, fracture risk calculators that integrate bone density measurements with clinical risk factors have shifted management of osteoporosis from an exclusive focus on bone density results to recommendations that incorporate absolute fracture risk. ${ }^{23}$

Communicating risk to patients is therefore fundamental to allow informed and 
shared decision-making. ${ }^{4}$ Problems that might arise when using estimates of the risk of a future event include misunderstanding numeric data and statistical concepts of risk and probability, both by the healthcare professional and the patient, and limited patient health literacy. ${ }^{5}{ }^{6}$ Research on communicating risks, benefits and harms to patients ${ }^{5-11}$ has generated evidence-based recommendations for communicating risk. ${ }^{6}$ They suggest that risks should be expressed as percentages or natural frequencies with benefits and harms expressed in absolute terms, supplemented by icon arrays, ${ }^{6}$ which can be presented using a decision aid. ${ }^{4}$

The framing of risk is influential in patient decisionmaking. Risk that is framed positively by description of benefits or gains is associated with less perception of harm and increased acceptance of therapies than when risk is framed negatively by use of harms or losses, ${ }^{6}$ but these differences may not greatly influence behaviour. ${ }^{9}$ Few trials have explored different approaches to communicating risk to patients for chronic conditions in which indications for treatment are based on the absolute risk of an event within a set time frame, such as cardiovascular disease and osteoporosis.

We set out to investigate the impact of communicating absolute risk in different ways to patients, using fracture as the model health event. We investigated the effects of framing of the risk of fracture and benefits of osteoporosis treatments, and whether this influences patients' beliefs about the need for osteoporosis treatment. In particular, we assessed the effect of presenting risk and treatment benefits in terms of percentages, numbers need to treat, or natural frequencies, and of framing risk differently (eg, a $5 \%$ chance of having a hip fracture vs a $95 \%$ chance of not having a hip fracture).

\section{METHODS}

We invited consecutive patients $>60$ years of age referred to a public hospital clinic for bone density measurement (October 2013 to July 2014) who were not taking any specific osteoporosis treatments to take part. Prior to their bone density measurement, consenting participants completed a questionnaire exploring their beliefs about their risk of fracture and the benefits they might obtain from treatment. Following the bone density measurement, the absolute risk of fracture within 5 years was calculated with the Garvan fracture risk calculator (http://www.garvan.org.au/promotions/bone-fracturerisk/calculator/). Participants were then randomised to receive one of four different written and pictorial presentations of their absolute fracture risk and the likely benefits they could expect from osteoporosis treatment. Group allocations were assigned by the study statistician using block randomisation with a variable block size schedule, based on computer-generated random numbers. Allocation concealment occurred through centralised randomisation. After reading the presentation, participants completed a second questionnaire about the risks of fracture and benefits of treatment. The bone density scan was reported in accordance with standard practice, including management recommendations based on the individual's absolute risk of fracture, and all participants were encouraged to discuss the report with their family doctor. We contacted all participants 3 months after their bone density scan to complete a third questionnaire exploring their beliefs about risk of fracture and the benefits of treatment. This study was registered at ANZCTR (ACTRN12613001081707).

\section{Questionnaires}

The three questionnaires are available in the online supplementary appendix. Briefly, we asked participants to rate their 5-year risks of having any fracture and having a hip fracture on a visual analogue scale of $0 \%$ (no chance) to $100 \%$ (definitely) and a scale of none to very high, and the 5-year total fracture and hip fracture risks of the average man or woman of the same age on a visual analogue scale of $0-100 \%$. We asked participants whether they thought they should take osteoporosis medication, questions exploring their reasons for taking medication or not, and to rate the effectiveness of osteoporosis treatments on a visual analogue scale of $0-100 \%$.

\section{Presentation of risk}

Table 1 shows the four presentations of absolute risk of fracture and treatment benefits, which were provided to the participants in writing. The first three sentences were identical for each group, and stated the participant's calculated 5-year risk of osteoporotic and hip fracture. Each randomised group then received text that framed risk either as the chance of having an event or not having an event within 5 years and with treatment benefits or the lack of treatment benefits (depending on the framing) in natural frequencies or presented as numbers needed to treat. All four options were accompanied by icon arrays depicting both the fracture risk and the treatment benefit (see online supplementary appendix).

\section{Statistics}

The prespecified primary analysis was a comparison between the four randomised groups of the perceived risks of total fracture and hip fracture at which treatment would be considered. Secondary end points were the perceived risk of fracture, and the perceived need for osteoporosis treatment. Since this was a novel study, it was difficult to estimate what effect sizes would be observed. Therefore, we pragmatically aimed to recruit 200 patients, on the basis that this was feasible within a timely period, and with 50 patients per group, the largest CI for a percentage result is $14 \%$ (for a proportion of $50 \%$ ). A difference of $\sim 20 \%$ could be detected in a pairwise comparison between two groups in this scenario. If the proportions were closer to $100 \%$ or $0 \%$, the CIs become 
Table 1 Text received by participants in each randomised group

Common text

Group 1: framed as chance of having an event and treatment benefits in natural frequencies

Group 2: framed as chance of not having an event and treatment benefits in natural frequencies

Group 3: framed as chance of having an event and treatment benefits as number needed to treat

Group 4: framed as chance of not having an event and treatment benefits as number needed to treat
- Based on the information in your questionnaire and your bone density:

- Your estimated risk of osteoporotic fracture (ie, all fractures except fractures of the skull, face, hands and feet) in the next 5 years is: $20 \%$.

- Your estimated risk of hip fracture in the next 5 years is: $5 \%$.

- This means that in a group of 100 people of the same age and gender as you, who had similar risk factors for fracture as you, 20 would have an osteoporotic fracture within the next 5 years, and 5 would have a hip fracture within the next 5 years.

- Osteoporosis medication reduces osteoporotic fractures by $33 \%$, and hip fractures by $40 \%$.

- This means that if all these 100 people took osteoporosis medication for 5 years, the number of people who would have an osteoporotic fracture within those 5 years would decrease from 20 to 13 . The number who would have a hip fracture within those 5 years would decrease from 5 to 3 .

- This means that in a group of 100 people of the same age and gender as you, who had similar risk factors for fracture as you, 80 will not have an osteoporotic fracture within the next 5 years, and 95 will not have a hip fracture within the next 5 years.

- Osteoporosis medication reduces osteoporotic fractures by $33 \%$, and hip fractures by $40 \%$.

- This means that if all these 100 people took osteoporosis treatments for 5 years, the number of people who would not have an osteoporotic fracture within those 5 years would increase from 80 to 87 . The number of people who would not have a hip fracture within those 5 years would increase from 95 to 97.

- This means that in a group of 100 people of the same age and gender as you, who had similar risk factors for fracture as you, 20 would have an osteoporotic fracture within the next 5 years, and 5 would have a hip fracture within the next 5 years.

- Osteoporosis medication reduces osteoporotic fractures by $33 \%$, and hip fractures by $40 \%$.

- This means that 15 people like you would need to be treated with osteoporosis medications for 5 years to prevent 1 osteoporotic fracture. 50 people like you would need to be treated for 5 years to prevent 1 hip fracture.

- This means that in a group of 100 people of the same age and gender as you, who had similar risk factors for fracture as you, 80 will not have an osteoporotic fracture within the next 5 years, and 95 will not have a hip fracture within the next 5 years.

- Osteoporosis medication reduces osteoporotic fractures by $33 \%$, and hip fractures by $40 \%$.

- This means that if 15 people like you were treated with osteoporosis medications for 5 years, 14 would receive no benefit in terms of osteoporotic fracture prevention, and in 1 person a fracture would be prevented. If 50 people like you were treated for 5 years, 49 would receive no benefit in terms of hip fracture prevention, and in 1 person a hip fracture would be prevented.

For illustrative purposes, all options use a 5 -year $20 \%$ risk of osteoporotic fracture and $5 \%$ risk of hip fracture.

narrower and the detectable differences become smaller. Since most data were non-normally distributed, we used non-parametric tests throughout, including the Kruskal-Wallis one-way analysis of variance test for comparisons between the four groups, and the signed-rank test for comparisons within groups. Spearman correlation analysis was used to test for significant associations between participant and calculator estimates of fracture risk. All tests were two-tailed and hypothesis tests were deemed significant for $\mathrm{p}<0.05$. $\mathrm{p}$ Values were not adjusted for multiple comparisons. All statistical analyses were carried out using the SAS software package (SAS Institute, Cary, North Carolina, USAV.9.4).

\section{RESULTS}

Two hundred people undergoing bone densitometry agreed to participate (see online supplementary appendix figure S1). Their baseline characteristics are shown in table 2 and were similar in the four randomised groups. The cohort is broadly representative of patients seen in clinical practice for bone densitometry; the average age was 69 years, $81 \%$ were female, $33 \%$ had a fracture after 50 years and the average femoral neck bone density $\mathrm{T}$ score was in the osteopenic range.

At baseline, the median (Q1, Q3) 5-year risk threshold participants regarded as high enough to consider taking medication to prevent any fracture was $50 \%(25,70)$ for 
oral tablets and $60 \%(30,80)$ for intravenous medication (table 3). For hip fracture, the respective 5-year risk thresholds were $50 \%(30,75)$ and $60 \%(40,80)$. The thresholds were similar in the four randomised groups. Figure 1 shows that providing the written estimates of fracture risk and treatment benefits led to no or very small changes in these risk thresholds (a decrease of $10 \%$ or less in all groups). There were no between-group differences in these changes $(p>0.6)$. At baseline, $46 \%$ of participants estimated that their hip or total fracture risk was equal to or greater than one of the thresholds they considered high enough to take preventative medication. After written information on fracture risk and treatment benefits was provided, $37 \%$ of participants estimated that their hip or total fracture risk was equal to or greater than one of the thresholds they considered high enough to take preventative medication.

At baseline, the median (Q1, Q3) 5-year risk of any fracture estimated by the participant was $20 \%(10,50)$, and for hip fracture was $19 \%(10,40$; table 3$)$. Sixty-one

Table 2 Baseline characteristics by randomised group

\begin{tabular}{lllll}
\hline & Group 1 & Group 2 & Group 3 & Group 4 \\
\hline $\mathrm{n}$ & 51 & 49 & 51 & 49 \\
Age (years) & $69.1(7.4)$ & $68.3(5.7)$ & $70.3(6.3)$ & $68.9(6.0)$ \\
Body mass index $\left(\mathrm{kg} / \mathrm{m}^{2}\right)$ & $26.7(5.1)$ & $27.1(4.7)$ & 75 & $26.5(5.4)$ \\
Female (\%) & 86 & 71 & 94 & 90 \\
European descent (\%) & 92 & 98 & 35 & 96 \\
Fracture after 50 years (\%) & 41 & 31 & $-0.4(2.0)$ \\
Bone mineral density T-score & $-0.5(1.6)$ & $-0.3(1.8)$ & $-1.1(1.2)$ \\
$\quad$ Lumbar spine & $-1.2(1.1)$ & $-0.9(1.3)$ & $-1.5(1.0)$ \\
$\quad$ Total hip & $-1.6(0.9)$ & $-1.3(1.1)$ & $-0.1(1.6)$ \\
$\quad$ Femoral neck & & $-1.1(2.3)$ \\
\hline $\begin{array}{l}\text { Data are per cent, or mean (SD). Group 1: framed as chance of having an event and treatment benefits in natural frequencies; group 2: } \\
\text { framed as chance of not having an event and treatment benefits in natural frequencies; group 3: framed as chance of having an event and }\end{array}$ \\
treatment benefits as number needed to treat; group 4: framed as chance of not having an event and treatment benefits as number needed to \\
treat.
\end{tabular}

Table 3 Influence of providing information communicating risk of fracture and treatment benefits

\begin{tabular}{|c|c|c|c|c|c|}
\hline & $\begin{array}{l}\text { Group } 1 \\
(n=51)\end{array}$ & $\begin{array}{l}\text { Group } 2 \\
(n=49)\end{array}$ & $\begin{array}{l}\text { Group } 3 \\
(n=51)\end{array}$ & $\begin{array}{l}\text { Group } 4 \\
(n=49)\end{array}$ & Entire cohort \\
\hline \multicolumn{6}{|c|}{ Participant estimates at baseline } \\
\hline \multicolumn{6}{|c|}{ 5-year fracture risk high enough to consider taking medications (tablet/intravenous) to prevent fracture (any/hip) (\%) } \\
\hline Any fracture/tablets (\%) & $50(23,75)$ & $50(28,60)$ & $50(35,80)$ & $50(20,60)$ & $50(25,70)$ \\
\hline Any fracture/intravenous (\%) & $53(40,80)$ & $55(20,72)$ & $60(50,80)$ & $60(30,80)$ & $60(30,80)$ \\
\hline Hip fracture/tablets (\%) & $50(30,80)$ & $50(35,73)$ & $55(50,80)$ & $48(20,60)$ & $50(30,75)$ \\
\hline Hip fracture/intravenous (\%) & $60(45,80)$ & $55(30,80)$ & $60(40,80)$ & $65(30,80)$ & $60(40,80)$ \\
\hline Risk of any fracture in next 5 years (\%) & $25(10,50)$ & $25(10,50)$ & $20(10,50)$ & $15(10,40)$ & $20(10,50)$ \\
\hline None/very low risk & 14 & 17 & 16 & 24 & 18 \\
\hline Low risk & 44 & 38 & 45 & 47 & 43 \\
\hline Moderate risk & 30 & 42 & 29 & 24 & 31 \\
\hline High risk & 12 & 4 & 10 & 2 & 7 \\
\hline Very high risk & 0 & 0 & 0 & 2 & 1 \\
\hline Risk of hip fracture in next 5 years (\%) & $15(10,50)$ & $20(10,45)$ & $20(10,40)$ & $10(5,30)$ & $19(10,40)$ \\
\hline \multicolumn{6}{|l|}{ Garvan fracture risk calculator estimates } \\
\hline 5-year osteoporotic fracture risk & $7.9(5.8,12.3)$ & $7.3(4.4,9.9)$ & $8.5(5.6,14.6)$ & $7.1(5.0,9.6)$ & $7.4(5.5,12.0)$ \\
\hline 5-year hip fracture risk & $1.6(0.8,3.4)$ & $1.2(0.7,2.4)$ & $1.8(0.8,4.1)$ & $1.3(0.6,2.0)$ & $1.4(0.8,3.0)$ \\
\hline \multicolumn{6}{|c|}{ Participant estimates after information on fracture risk and treatment benefits provided } \\
\hline Risk of any fracture in next 5 years (\%) & $14(9,30)$ & $19(10,27)$ & $15(7,30)$ & $10(8,20)$ & $12(8,30)$ \\
\hline None/very low risk & 20 & 29 & 27 & 33 & 27 \\
\hline Low risk & 45 & 47 & 47 & 47 & 47 \\
\hline Moderate risk & 31 & 18 & 20 & 10 & 20 \\
\hline High risk & 4 & 6 & 4 & 8 & 6 \\
\hline Very high risk & 0 & 0 & 2 & 2 & 1 \\
\hline Risk of hip fracture in next 5 years (\%) & $8(2,20)$ & $10(2,20)$ & $10(2,20)$ & $10(2,15)$ & $10(2,20)$ \\
\hline
\end{tabular}


Figure 1 Box and whisker plots of the changes in the 5-year risk thresholds participants considered high enough to take treatment, either by tablets or by intravenous infusion, to prevent any fracture or hip fracture after written information on fracture risk and treatment benefits was provided by treatment group. Group $1(n=51)$ : framed as chance of having an event and treatment benefits in natural frequencies; group $2(n=49)$ : framed as chance of not having an event and treatment benefits in natural frequencies; group 3 $(n=51)$ : framed as chance of having an event and treatment benefits as number needed to treat; group $4(n=49)$ : framed as chance of not having an event and treatment benefits as number needed to treat.

per cent considered that their risk of any fracture was low or very low. The median 5-year risk of any fracture estimated by the participants for an average man or woman of the same age was $40 \%(20,50)$ and for hip fracture was $30 \%(20,50)$. Fifty-nine per cent of participants estimated that their individual risk of any fracture was lower than that of the average person, and $67 \%$ estimated that their hip fracture risk was lower than average. Table 3 and figure 2 show that the estimated risks of fracture by the participants and from the Garvan calculator were similar for the randomised groups. For the entire cohort and for each randomised group, the 5-year risk of total fracture estimated by the participants was 2-3 times higher than the calculator estimates $(\mathrm{p}<0.001$ for all groups). For hip fracture, the participant estimates were only slightly lower than the estimates for any fracture and were 10-20 times higher than the calculator estimates $(p<0.001$ for all groups). The correlation between participant and calculator risk estimates was modest: $\mathrm{r}=0.33, \mathrm{p}<0.001$ for any fracture and $\mathrm{r}=0.22, \mathrm{p}=0.002$ for hip fracture.

Table 3 and figure 2 show the influence of providing information on the individual participant's fracture risk from the Garvan calculator on participants' estimates of their own fracture risk. There were small (0-10\%) reductions in participants' perceptions of their total and hip fracture risk that were not different between groups ( $p=0.50$ for total fracture risk, $p=0.42$ for hip fracture risk). In all groups, the participant estimates remained much higher than the calculator estimates after these estimates were provided to the participants $(p<0.001$ for all groups).

Prior to their bone density scan, $15 \%$ of participants felt they should take medication to prevent fractures, $34 \%$ felt they should not take medication and $51 \%$ were unsure (table 4, see online supplementary appendix table S1). The proportions did not change substantially after the calculator estimates and treatment benefits were provided-the respective proportions were $19 \%$, $51 \%$ and $30 \%$. At 3 months after the bone density scan, $34 \%$ of participants indicated that they had started medication or were intending to. A similar proportion (43-48\%) of participants who felt they should or should not take osteoporosis medication, or did not know at baseline, estimated that their hip or total fracture risk was equal to or greater than one of the thresholds they considered high enough to take preventative medication (see online supplementary appendix table $\mathrm{S} 1$ ).

Table 4 shows that one-third of those who believed they should take osteoporosis medication before their bone density measurement changed their views after receiving the information on fracture risk and treatment benefits, and a similar proportion had not started or did not intend to start osteoporosis medication at the 3-month follow-up. Of those who initially believed they should not take osteoporosis medication, $80 \%$ persisted with that belief after receiving the information on fracture risk and treatment benefits, and a similar proportion had not started or did not intend to start osteoporosis medication at the 3-month follow-up. Of the group that was undecided initially, about half remained undecided after receiving the information on fracture risk and treatment benefits, but one-third had started or intended to start medication at the 3-month follow-up.

\section{DISCUSSION}

In this study, providing estimated absolute risks of fracture and benefits of treatment in four different ways had 
Figure 2 Box and whisker plots of the estimated 5-year risk of osteoporotic and hip fracture before and after the provision of fracture risk estimates from the Garvan calculator. Group 1 $(n=51)$ : framed as chance of having an event and treatment benefits in natural frequencies: group $2(n=49)$ : framed as chance of not having an event and treatment benefits in natural frequencies; group $3(n=51)$ : framed as chance of having an event and treatment benefits as number needed to treat; group 4 $(n=49)$ : framed as chance of not having an event and treatment benefits as number needed to treat.
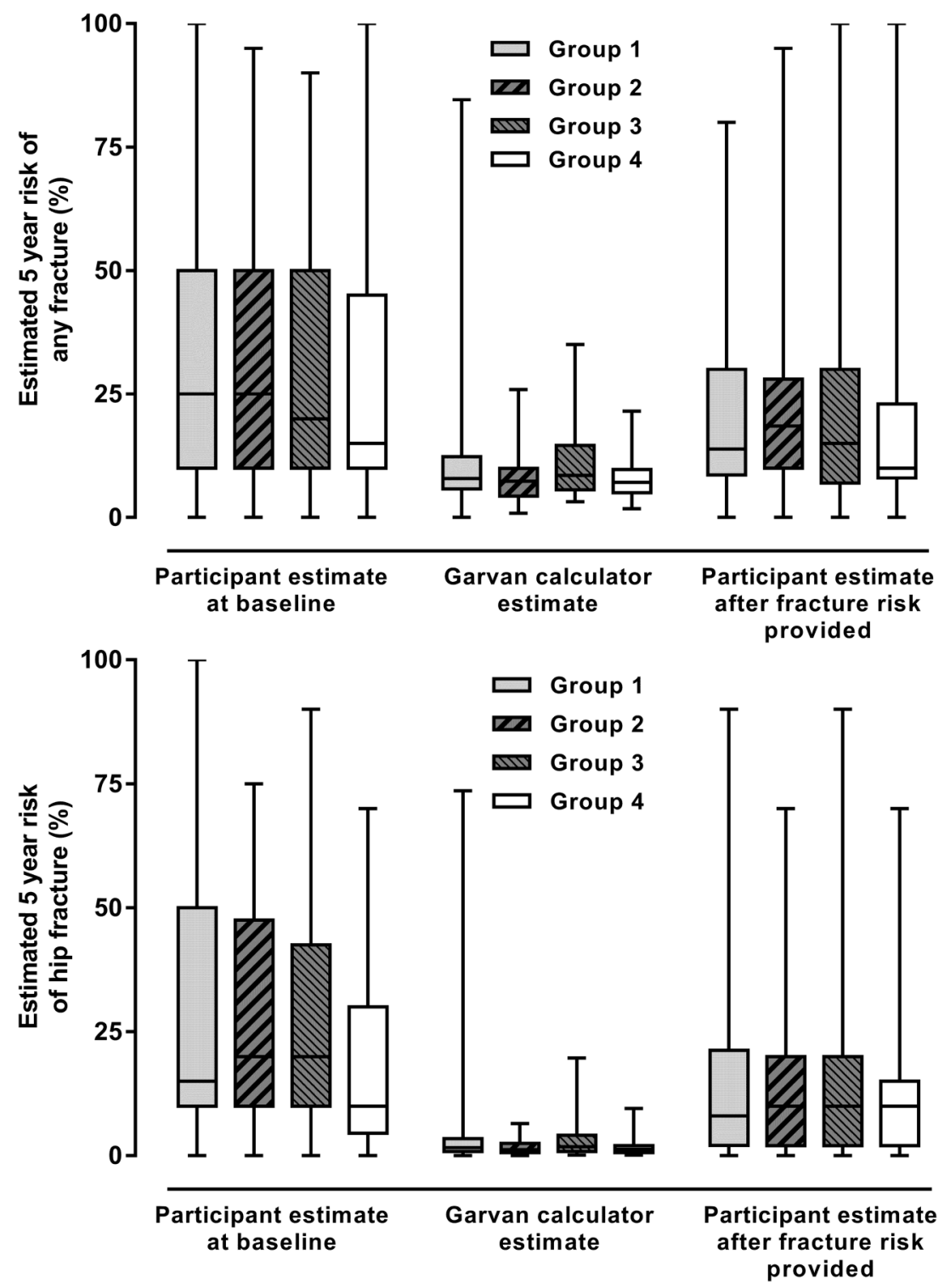

Table 4 Participant views about taking osteoporosis medicine grouped by their initial views

\begin{tabular}{|c|c|c|c|}
\hline & \multicolumn{3}{|c|}{$\begin{array}{l}\text { Participants' initial view on } \\
\text { whether they should take } \\
\text { osteoporosis medication }\end{array}$} \\
\hline & Yes & No & Don't know \\
\hline \multicolumn{4}{|c|}{ Prior to bone density scan } \\
\hline n (\%) & $30(15)$ & $67(34)$ & $101(51)$ \\
\hline \multicolumn{4}{|c|}{$\begin{array}{l}\text { After information on fracture risk and treatment benefits } \\
\text { provided }\end{array}$} \\
\hline \multicolumn{4}{|c|}{ Should you take osteoporosis medication? } \\
\hline Yes (\%) & $20(67)$ & $3(4)$ & $14(14)$ \\
\hline No $(\%)$ & $8(27)$ & $54(81)$ & 39 (39) \\
\hline Don't know (\%) & $2(7)$ & $10(15)$ & $48(48)$ \\
\hline \multicolumn{4}{|c|}{ At 3-month follow-up } \\
\hline \multicolumn{4}{|c|}{ Started/intend to start osteoporosis medication } \\
\hline Yes (\%) & $17(65)$ & $12(19)$ & $34(35)$ \\
\hline No $(\%)$ & $9(35)$ & $51(81)$ & $62(65)$ \\
\hline
\end{tabular}

little effect on participants' perception of their need to take treatment or their individual risk of fracture. Previous research and trials on risk communication have generally reported important differences between presentations of natural frequencies or numbers needed to treat and between different framing styles, but these studies mainly focused on understanding of risk rather than need for intervention. ${ }^{5}$ 8-10 $^{8}$ Before their bone density scan, the average 5-year fracture risk threshold at which participants would consider treatment was 50$60 \%$. These thresholds changed little after information on fracture risk and treatment benefits was provided. Prior to receiving this information, participants overestimated their risk of any fracture by 2-3 times and of hip fracture by 10-20 times. After receiving a written description of their fracture risk, participants' estimates of their risk of fracture halved but remained 1.5-2 times higher than the Garvan estimates for any fracture, and 5-10 times higher for hip fracture. Framing the 
presentation of risk as the chance of having a fracture did not produce different results from framing the presentation as the chance of not having a fracture.

\section{Strengths and limitations}

The strengths of the study are its randomised allocation to different methods of risk communication and relevance to clinical practice. Although there is a large body of research into risk communication, ${ }^{5-11}$ we are not aware of similar trials that have explored the impact of risk framing on risk perception and treatment benefits, either where the absolute risk of an event forms the basis for treatment recommendations or in the field of osteoporosis. Participants were patients undergoing standard clinical care, so the results may be generalisable to similar outpatient populations. There are limitations to our results. Our cohort was of moderate size and had a relatively low fracture risk. Whether the findings would be similar in cohorts at a higher or lower risk of fracture, in cohorts that were not undergoing bone densitometry, or in other conditions, such as cardiovascular disease, is worth exploring. The questionnaires, presentation of results and icon arrays were designed for this study, and different results might be obtained using different text or icon arrays, or if similar information is discussed within the context of a clinical consultation. The results at 3 months will most likely be influenced by the bone density report and the views of the primary care doctor.

\section{Comparison to other studies}

Previously, we reported that a group of patients surveyed prior to bone density measurement substantially overestimated their individual risk of fracture,${ }^{12}$ findings similar to those from the current study. Other studies that have reported participants' views on their fracture risk found that older women generally consider themselves to be at lower risk of fracture than their peers, as we found in this study. The GLOW study reported that $43-49 \%$ of women (mean age 69 years) felt their fracture risk was below average, with only $12-15 \%$ considering themselves above average risk. ${ }^{13}$ Likewise in the ROSE study, $42 \%$ of women (mean age 71 years) considered that their risk was below average, and only $5 \%$ considered their risk was above average. ${ }^{14}$ There was a poor correlation between participants' own estimate of their 10-year fracture risk and the estimate from the FRAX calculator. ${ }^{14}$ However, participants were invited to classify their risk into five categories $(<10 \%, 10-14 \%, 15-19 \%, 20-24 \%$ and $\geq 25 \%)$ and by providing these values, the investigators may have introduced an anchoring bias into participant estimates. Collectively, the results suggest that people have an optimistic bias about their personal risk, ${ }^{15}$ generally considering themselves healthier and at lower risk than the average person. Nevertheless, their numeric estimates of their risk are substantial overestimates.

One previous trial ${ }^{16}$ randomised participants with low bone density to receive standard care or a decision aid that contained written descriptions of fracture risk and treatment benefits: the aid improved understanding of these concepts. However, consistent with the findings from our study, $51 \%$ of women who used the aid and $72 \%$ of women receiving standard care were unable to correctly identify their fracture risk from three categories $(<10 \%, 10-30 \%$ and $>30 \%)$. Taken together, the results of these studies suggest that patients have difficulty understanding information about risk presented in written and pictorial formats and that research is required into what patients think an absolute risk of fracture represents.

More broadly, previous studies on framing of risk reported that positive framing led to a better understanding of the message, and higher ratings of perceived effectiveness of therapies than negative framing. ${ }^{9}$ However, other studies reported that framing did not appear to affect hypothetical decisions or intentions to adopt interventions, or actual behaviour. ${ }^{9}$ In our study, framing of risk had little effect on patients' perceived fracture risk or views about treatment, consistent with the latter studies. Previous studies reported that the use of absolute risk reductions is better understood than the use of numbers needed to treat, is associated with higher ratings of perceived effectiveness, but is not associated with differences in effects on hypothetical decisions or intentions to adopt interventions. ${ }^{8}$ In our study, the use of absolute risk reductions presented with natural frequencies did not alter patients' perceived fracture risk or views about treatment compared with the use of numbers needed to treat.

\section{Study meaning and interpretation}

Some of our findings are surprising. We anticipated participants overestimating their risk of fracture at baseline. ${ }^{12}$ However, we expected that after being provided with an explicit description of their estimated fracture risks, participants would align their personal estimates of fracture risk with the provided values. Failure to do so suggests that the participants either did not understand the concept of risk or the presentation of results, or they did not believe the estimates provided.

The results highlight some interesting features of risk perceptions among participants undergoing bone densitometry. At baseline, the median 5-year risk of any fracture estimated by participants was $20 \%$, and for hip fracture was $19 \%$. It is not clear whether participants therefore believe that non-hip fractures are extremely rare, or that they misunderstood the question or answer. Both of these levels of risk would be categorised as high by most osteoporosis guidelines, ${ }^{2}{ }^{3}$ yet only $7-8 \%$ of participants viewed their risk as high or very high and $61 \%$ considered their risk as low or very low. The median thresholds of 5-year fracture risk at which participants considered they would take preventative medication were $40-60 \%$ and $46 \%$ of participants' own estimates of fracture risk were equal to or greater than these thresholds. However, this seemed unrelated to the decision to 
take treatment: similar proportions $(43-48 \%)$ of people whose own estimates of fracture risk were greater than or equal to their own treatment thresholds believed they should or should not take osteoporosis medication or did not know. Similar to the findings of our previous survey, ${ }^{12}$ these contradictions highlight large and important discrepancies between patients' and healthcare professionals' perception of fracture risk, and intervention thresholds.

Undertaking a bone density scan tended to reinforce rather than change patients' views about the need for treatment. Thus, only $35 \%$ of people who believed that they should take osteoporosis medication before the scan and $19 \%$ of people who believed that they should not take osteoporosis medication changed their views 3 months after the scan. The small differences between these two groups in bone density, participants' estimated fracture risk and Garvan risk estimates, are unlikely to explain the differing perceptions about the perceived need for medication. For the majority of people with a view about the need for osteoporosis medication before having a bone density scan, the results of the scan appear to have confirmed their pre-existing beliefs, regardless of the result. This may represent a confirmation bias, whereby attention is focused on aspects of the results that support the pre-existing beliefs while aspects that challenge the beliefs are downplayed or ignored. ${ }^{17}$

The majority of participants in the study considered that they were at lower risk of fracture than average, a consistent finding in many studies termed the 'better than average effect'. Providing comparisons of risk to the average person can change risk perception. ${ }^{18}$ Individuals who believe that they are at lower risk than average may consider that they do not need to take treatment without actually considering the benefits of the treatment.

In summary, we found that patients referred for bone densitometry have a high threshold of fracture risk before they would consider taking treatment to prevent fractures, and this does not change after written information on fracture risk and treatment benefits is provided. These patients also substantially overestimate their risk of fracture, even after fracture risk estimates are provided to them explicitly in writing. We identified a number of logical contradictions in patients' views about fracture risk that present challenges for healthcare practitioners trying to accurately communicate fracture risk to patients as the first step in allowing informed, shared decision-making. It seems unwise to assume that simply providing absolute risks of fracture and treatment benefits to patients is adequate to allow this to occur. It is important to explore whether these findings are specific to fracture risk, or are a more general feature of conditions where absolute risk estimates of health events are a fundamental component, such as cardiovascular disease.

Contributors RK, KJP, AG, GDG and MJB designed the research. RK, ZN, $A M H$ and MJB ran the trial. MJB and GDG performed the analyses. MJB drafted the paper. All authors critically reviewed and improved it. MJB is the guarantor for the article. All authors had access to all the data and take responsibility for the integrity of the data and the accuracy of the data analysis.

Funding Funded by the Health Research Council (HRC) of New Zealand. The authors are independent of the HRC.

Competing interests All authors have completed the ICMJE uniform disclosure form at http://www.icmje.org/coi_disclosure.pdf and declare: the study was funded by the Health Research Council (HRC) of New Zealand. AG is a shareholder in Auckland Bone Density, an organisation that provides bone densitometry services.

Ethics approval Northern A Health and Disability Ethics Committee.

Provenance and peer review Not commissioned; externally peer reviewed.

Data sharing statement Patient-level data are available from the corresponding author on reasonable request. The presented data are anonymised and risk of identification is low.

Open Access This is an Open Access article distributed in accordance with the Creative Commons Attribution Non Commercial (CC BY-NC 4.0) license, which permits others to distribute, remix, adapt, build upon this work noncommercially, and license their derivative works on different terms, provided the original work is properly cited and the use is non-commercial. See: http:// creativecommons.org/licenses/by-nc/4.0/

\section{REFERENCES}

1. Jackson R. Guidelines on preventing cardiovascular disease in clinical practice. BMJ 2000;320:659-61.

2. Dawson-Hughes B. A revised clinician's guide to the prevention and treatment of osteoporosis. J Clin Endocrinol Metab 2008;93: 2463-5.

3. Compston J, Cooper A, Cooper C, et al. Guidelines for the diagnosis and management of osteoporosis in postmenopausal women and men from the age of 50 years in the UK. Maturitas 2009;62:105-8.

4. McCartney M, Treadwell J, Maskrey N, et al. Making evidence based medicine work for individual patients. BMJ 2016;353:i2452.

5. Gigerenzer G, Edwards A. Simple tools for understanding risks: from innumeracy to insight. BMJ 2003;327:741-4.

6. Zipkin DA, Umscheid CA, Keating NL, et al. Evidence-based risk communication: a systematic review. Ann Intern Med 2014;161:270-80

7. Ancker JS, Senathirajah Y, Kukafka R, et al. Design features of graphs in health risk communication: a systematic review. J Am Med Inform Assoc 2006;13:608-18.

8. Akl EA, Oxman AD, Herrin J, et al. Using alternative statistical formats for presenting risks and risk reductions. Cochrane Database Syst Rev 2011;(3):CD006776.

9. Akl EA, Oxman AD, Herrin J, et al. Framing of health information messages. Cochrane Database Syst Rev 2011;(12):CD006777.

10. Ahmed $\mathrm{H}$, Naik $\mathrm{G}$, Willoughby $\mathrm{H}$, et al. Communicating risk. BMJ 2012;344:e3996.

11. Stacey D, Bennett CL, Barry MJ, et al. Decision aids for people facing health treatment or screening decisions. Cochrane Database Syst Rev 2011;(10):CD001431.

12. Douglas F, Petrie KJ, Cundy T, et al. Differing perceptions of intervention thresholds for fracture risk: a survey of patients and doctors. Osteoporos Int 2012;23:2135-40.

13. Jones SM, Gell NM, Roth JA, et al. The relationship of perceived risk and biases in perceived risk to fracture prevention behavior in older women. Ann Behav Med 2015;49:696-703.

14. Rothmann MJ, Ammentorp J, Bech M, et al. Self-perceived facture risk: factors underlying women's perception of risk for osteoporotic fractures: the Risk-Stratified Osteoporosis Strategy Evaluation study (ROSE). Osteoporos Int 2015;26:689-97.

15. Weinstein ND. Optimistic biases about personal risks. Science 1989;246:1232-3.

16. Montori VM, Shah ND, Pencille LJ, et al. Use of a decision aid to improve treatment decisions in osteoporosis: the osteoporosis choice randomized trial. Am J Med 2011;124:549-56.

17. Nickerson RS. Confirmation bias: a ubiquitous phenomenon in many guises. Rev Gen Psychol 1998;2:175-220.

18. Fagerlin A, Zikmund-Fisher BJ, Ubel PA. "If I'm better than average, then I'm ok?": comparative information influences beliefs about risk and benefits. Patient Educ Couns 2007;69:140-4. 